\title{
Allergologie quasi olympisch
}

들 Herausgeber, 41 zusätzliche Autoren, 592 Seiten, $285 \times 220 \times 32 \mathrm{~mm}$ Außenmaße und fast $2 \mathrm{~kg}$ schwer ... das „Handbuch“ im Titel ist vornehme $\mathrm{Zu}$ rückhaltung oder ein Versehen des Verlages. Die Veröffentlichung eines derartig profunden Fachbuchs zum Thema Allergologie war bisher ein angelsächsisches Privileg. Vergessen Sie jetzt Ihr Schulenglisch und mobilisieren Sie die Reserven vom letzten weihnachtlichen Kaufrausch.

Experten ihres Faches stellen in diesem Werk Grundlagen, Diagnostik, Krankheitsbilder und Therapiestrategien der Allergologie vor, so dass nicht nur Anfänger, sondern auch alte Hasen auf ihre Kosten kommen. Den Autoren ist der Spagat gelungen: Dies ist nicht nur ein systematisches, reich gegliedertes Lehrbuch mit 13 Seiten Inhaltsverzeichnis (sportlich!), sondern auch eine Fundgrube für praktische Perlen und delikate Details. Literaturhinweise im Text weisen den Weg zu den Origi- nalquellen, die allerdings in manchen Kapiteln etwas sparsam zitiert worden sind. Gerade hier entsteht für den wissenschaftlich interessierten Leser echter Mehrwert; besonders wenn es um Quellen geht, die nicht ohne Weiteres im großen, virtuellen Netz abgeschöpft werden können.

Das Fachbuch hat es heute ohnehin schwer: Die Halbwertszeit unseres Wissens verringert sich kontinuierlich, viele verlassen sich daher eher auf Periodika oder das exponenziell wachsende Internet als Informationsquelle. Aber dennoch, ein übersichtliches, deutschsprachiges Spezialbuch mit gefiltertem Expertenwissen zu sämtlichen Aspekten der Allergologie wird am ehesten dem Querschnittscharakter unserer Lieblingsdisziplin gerecht und ist lange Zeit überfällig gewesen.

Die einheitliche Aufbereitung des Stoffes, liebevoll gestaltete Grafiken, zahlreiche Textkästen und Tabellen, ausgezeichnetes fotografisches Bildma- terial und didaktisch hilfreiche Merksätze verführen zum Stöbern. Gerne liest man sich fest, egal ob es um Allergenkunde, klinische Spezialitäten, diagnostische Empfehlungen oder Therapieinnovationen geht. Offenbar war die Symbiose zwischen Herausgebern, Autoren und Verlag erfolgreich: Fachlich und inhaltlich einwandfreie Beiträge wurden professionell zusammengefügt und für den Leser hochwertig aufbereitet. Das dargestellte theoretische und praktische Wissen eignet sich sowohl zum Einstieg in die Allergologie als auch als Nachschlagewerk für Spezialfragen. Dem Buch sei eine weite Verbreitung gewünscht.

Priv.-Doz. Dr. Jörg Kleine-Tebbe, Berlin

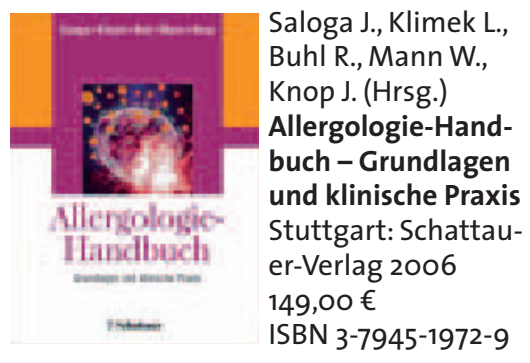

